\title{
Polar observations of ion/electron bursts at the pre-dawn polar cap boundary: evidence for internal reconnection of overdraped lobe flux
}

\author{
P. E. Sandholt ${ }^{1}$ and C. J. Farrugia ${ }^{2}$ \\ ${ }^{1}$ Department of Physics, University of Oslo, Oslo, Norway \\ ${ }^{2}$ Space Science Center, University of New Hampshire, Durham
}

Received: 30 January 2008 - Revised: 5 June 2008 - Accepted: 3 July 2008 - Published: 4 August 2008

\begin{abstract}
Observations made by Polar of ion-electron bursts on the dawn side of the polar cap are presented. They occurred when conditions external to the magnetosphere corresponded to that of the sheath region of a magnetic cloud, which was characterized by very high densities/dynamic pressure and a magnetic field which was strong in all components and which was tilted antisunward $\left(B_{x}<0\right)$ and northward $\left(B_{z}>0\right)$ with its clock angle lying between 20 and $90^{\circ}$ ( $\left.B_{y}: 8-15 \mathrm{nT}\right)$. A clear temporal development in the energy range spanned by the individual ion bursts (from $0.2-2 \mathrm{keV}$ to $1-10 \mathrm{keV}$ ) was present. We relate this to a corresponding temporal evolution in the cloud sheath field and plasma. We analyze the solar wind-magnetosphere aspects of the observations using the concepts of (i) (i) overdraped lobe flux, (ii) $B_{x}$ - and $B_{y}$-regulated sequential reconnections in opposite hemispheres (magnetopause and internal modes), and (iii) newly-closed magnetic flux. In particular, we find that the most energetic ion bursts (accompanied by bi-directionally streaming electrons at $1-10 \mathrm{keV}$ and intense magnetosheathorigin fluxes) are located on newly closed field lines generated by internal reconnection occurring between overdraped lobe field lines and the closed geomagnetic field. This result corroborates a topology of lobe reconnection under conditions of dipole tilt and/or nonzero IMF $B_{x}$ component advanced by Watanabe et al. (2006), which in our case is adapted to nonzero IMF $B_{y}$ conditions.
\end{abstract}

Keywords. Ionosphere (Plasma convection) - Magnetospheric physics (Magnetopause, cusp, and boundary layers; Solar wind-magnetosphere interactions)

Correspondence to: P. E. Sandholt

(p.e.sandholt@fys.uio.no)

\section{Introduction}

In this contribution to studies of magnetospheric reconnection under northward IMF conditions, we analyze a sequence of energetic ion bursts and associated electron flux enhancements detected by the Polar spacecraft in the pre-dawn northern magnetospheric lobe at mid-altitudes. We relate the plasma and magnetic field observations from Polar to recent ideas on the interconnection topology, particularly to the concept of "internal reconnection" discussed by Crooker (1992), and the associated magnetic flux circulation (see also Alexeev et al., 2008, and references therein). A variant of "internal reconnection" (so called because it occurs Earthward of the magnetopause), with a different flux circulation, was recently formulated by Watanabe et al. (2004) and Watanabe et al. (2006).

Reconnection of the magnetosheath and magnetospheric fields under a northward-pointing IMF leads to a variety of possible topologies which preclude a straightforward description as they are sensitively dependent on the dipole tilt (season), the $B_{x}$ component of the IMF and even moderate deviations of the IMF in the GSM Y-Z plane as measured by the clock angle. Central to these studies is the concept of "overdraped lobe flux" introduced by Crooker (1992), based on the idea of a two-step, sequential lobe reconnection occurring in alternate hemispheres first discussed by Cowley (1981). According to Cowley and Crooker the magnetic flux cycle starts with lobe reconnection in the hemisphere favoured by dipole tilt (summer) and/or IMF $B_{x}$ polarity (the first step). The newly opened lobe flux, which drapes over the dayside closed field line region, then undergoes a second reconnection, this time with open lobe flux of the opposite hemisphere. The newly closed lobe flux thereafter partakes in the convection cycle and is transported to the nightside. The convection cycle associated with this dual lobe reconnection consists of so-called twin "reverse" convection cells (sunward flow over the central polar cap), whose existence

Published by Copernicus Publications on behalf of the European Geosciences Union. 
has recently been confirmed by radar observations during intervals of strongly northward IMF conditions (clock angle $<10-20^{\circ}$ ) (Chisham et al., 2004; Imber et al., 2006).

An important variant of this cycle was recently proposed by Watanabe et al. (2006) and concerns the second step. In this variant, the overdraped lobe flux can reconnect with closed magnetospheric field lines Earthward of the magnetopause. Furthermore, in the presence of a nonzero east-west IMF component, $B_{y}$, the "internal reconnection" may occur on the dawn and dusk sides. It gives rise to a magnetic flux circulation cycle that differs from the Cowley/Crooker cycle.

Inspired by the MHD simulation study of Tanaka (1999) Watanabe et al. (2004) suggested that the inclusion of a nonzero IMF $B_{y}$ component can give rise to a a different first step in the two-step sequential reconnections at the magnetopause and inside the magnetosphere: magnetopause reconnection between the IMF and the $B_{y}$ - distorted Earth field taking place equatorward of the cusp. This gives rise to two types of overdraped lobe flux, those resulting from the northern (the traditional NA fluxes) and those resulting from the Southern Hemisphere (the nontraditional NB fluxes) magnetopause reconnections. For $B_{y}>0$ (our case), the second step (internal reconnection of the NB type overdraped lobe flux in the Southern Hemisphere) creates newly open and newly closed lobe fluxes on the dawn and dusk sides of the polar cap, respectively (see illustration in Fig. 4 of Watanabe et al., 2004). A fundamental difference between the Watanabe et al. (2004) and the Watanabe et al. (2006) models which we shall apply in this study is that newly closed flux is created on the dawn side in Watanabe et al. (2006) whereas it appears on the opposite (dusk) side in the Watanabe et al. (2004) model. The Watanabe et al. (2004) model creates two specific crescentshaped convection cells at either side of the polar cap referred to as primary and secondary exchange cells (PE and SE). The favourable IMF condition for the excitation of these cells is northeast/west orientation (clock angle near $45^{\circ}$ ).

In the case we study (12 August 2000), the external conditions are those of the sheath region of a magnetic cloud. Over a two-hour period (00:00-02:15 UT), the sheath field changed its orientation slowly while remaining in the clock angle range $20-90^{\circ}\left(B_{y}: 8-15 \mathrm{nT}\right)$. There were subintervals with clock angle near $45^{\circ}$, which are thus ideal to test predictions on internal reconnection in the oblique northward field case, particularly in view of Polar's footpoint position at the predawn polar cap boundary, as we shall describe below. Lobe reconnection in the Northern Hemisphere is favoured by dipole tilt/season and the prevailing negative $B_{x}$ component of the cloud sheath.

Applying the Polar observations of ion-electron bursts and the associated ion drift data we consider three different predicted scenarios for excitation of newly closed and/or newly open fluxes on the dawn side: (i) the newly-open lobe flux of Watanabe et al. (2004) and the associated SE convection cell, or (ii) the newly-closed lobe flux and the reverse convection cells produced by the internal reconnection of the Cowley-
Crooker-Alexeev type (overdraped lobe flux reconnnecting with open lobe flux), or (iii) the newly-closed lobe flux excited by reconnection of the overdraped lobe flux and the closed field of the Earth as suggested by Watanabe et al. (2006).

Based on the magnetic deflections and ion drifts accompanying the ion-electron bursts detected by Polar, a link to "post-terminator FTEs" (Kawano and Russell, 1997) is established. The "post-terminator" designation was introduced as a way of saying that pulsed reconnection (FTEs) may occur not only on the dayside (as the extensive ISEE data sets showed and from which FTEs were discovered) but also on the nightside (pre-dawn and post-dusk).

The paper is organized as follows. First we give a schematic description of the internal reconnection models of Watanabe et al. (2004), Watanabe et al. (2006) and Alexeev et al. (2008) which may all be potentially applicable to our $B_{x}<0, B_{y}>0$, and $B_{z}>0$ case. Then we report the interplanetary observations from WIND and magnetospheric observations acquired by spacecraft Polar. This is followed by discussion and conclusion.

\section{Model presentations}

Figure 1 shows a sketch of the magnetospheric topology for northward IMF orientation, with X-lines in the Northern and Southern Hemispheres, adapted to our case (IMF $B_{x}<0$; $B_{y}>0 ; B_{z}>0$ ) after Watanabe et al. (2006). The position of spacecraft Polar (at two times) is marked by starred symbols. During the interval we study Polar is situated within two hours of the dawn meridian (04:00-06:00 MLT), ascending in invariant latitude (ILT) and descending in altitude. The positions of Polar in GSM $(\mathrm{X}, \mathrm{Z})$ coordinates at 00:00 and $02: 00 \mathrm{UT}$ are $(-1.7,6.23)$ and $(-0.16,6.24) R_{E}$, respectively. The stages of a magnetospheric flux cycle are indicated in the figure in terms of numbered field lines: (1) newly reconnected field lines resulting from lobe reconnection in the north (in the postnoon sector), (2-3) overdraped lobe flux convecting dawnward (out of the plane of the paper), (4) newly-closed and (5) newly opened lobe flux after internal reconnection in the Southern Hemisphere with closed magnetospheric flux (4') in the pre-noon/dawn sector (downstream of the cusp), (6) "relaxed" open lobe flux and (7) detached flux in the midnight sector. This flux circulation model (1-7) corresponds to the C-D cycle and the associated merging cell called M2 by Watanabe et al. (2006) (see their Fig. 2). Types $C$ and $D$ reconnections are those taking place at the high-latitude magnetopause in the north and the internal reconnection in the south, respectively. Type $\mathrm{C}$ reconnection converts nightside "relaxed" lobe flux (6) to dayside overdraped lobe flux (1-2-3). Type D reconnection converts dayside overdraped lobe flux (3) to nightside relaxed lobe flux (6). Thus, type $\mathrm{C}$ reconnection creates overdraped lobe flux while the type $\mathrm{D}$ reduces the overdraped lobe flux. 


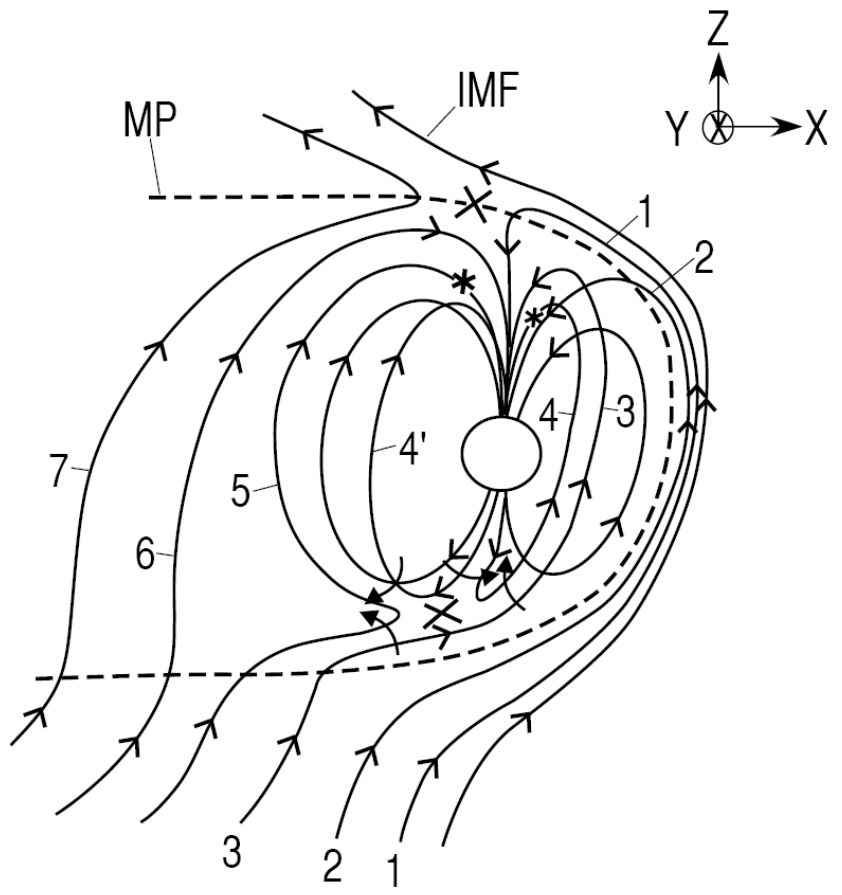

Fig. 1. Schematic illustration of magnetospheric magnetic flux cycle involving (i) high-latitude (lobe) reconnection in the north, (ii) overdraped lobe flux and (iii) internal reconnection between the overdraped lobe flux and closed geomagnetic field lines in the south at the dawn side during strongly north IMF orientation (the IMF $B_{x}<0$ case). The perspective is from the dawn side of the magnetosphere. Positions (at two times) of spacecraft Polar at mid-altitudes on the dawn side in relation to the field line topology are indicated by stars. Adapted after Watanabe et al. (2006). Not to scale.

However, not all of the type $\mathrm{C}$ reconnection is coupled to the type $\mathrm{D}$ reconnection. The uncoupled part of type $\mathrm{C}$ reconnection forms a self-contained magnetic flux circulation which is referred to as lobe cell circulation (1-2-6). The model discussed above is applicable to conditions of strongly north IMF orientation and significant dipole tilt/IMF $B_{x}$ component. In Fig. 1, adapted to our case, we take into account a positive IMF $B_{y}$ component as well. Plasma injections onto newly-closed (4) and newly-open (5) lobe fluxes during the internal reconnection (type D) are indicated in the figure by the solid arrowed lines. The approximate position of spacecraft Polar in relation to these fluxes at different times of our case interval are also indicated by starred symbols. We note that the indicated overdraped lobe flux on the dawn side for $B_{z}>0, B_{y}>0$ and $B_{x}<0$ conditions (field line 3 in the figure) is consistent with the recent model calculations of Alexeev et al. (2008) (see their Figs. 6-8).

A sketch of the ionospheric footprints of the convection cycles described above is given in Fig. 2. This convection pattern is adapted to our case after Fig. 2 of Watanabe et al. (2005). Footprint trajectories of spacecraft Polar and DMSP F12 are indicated. The F12 data documents the presence of

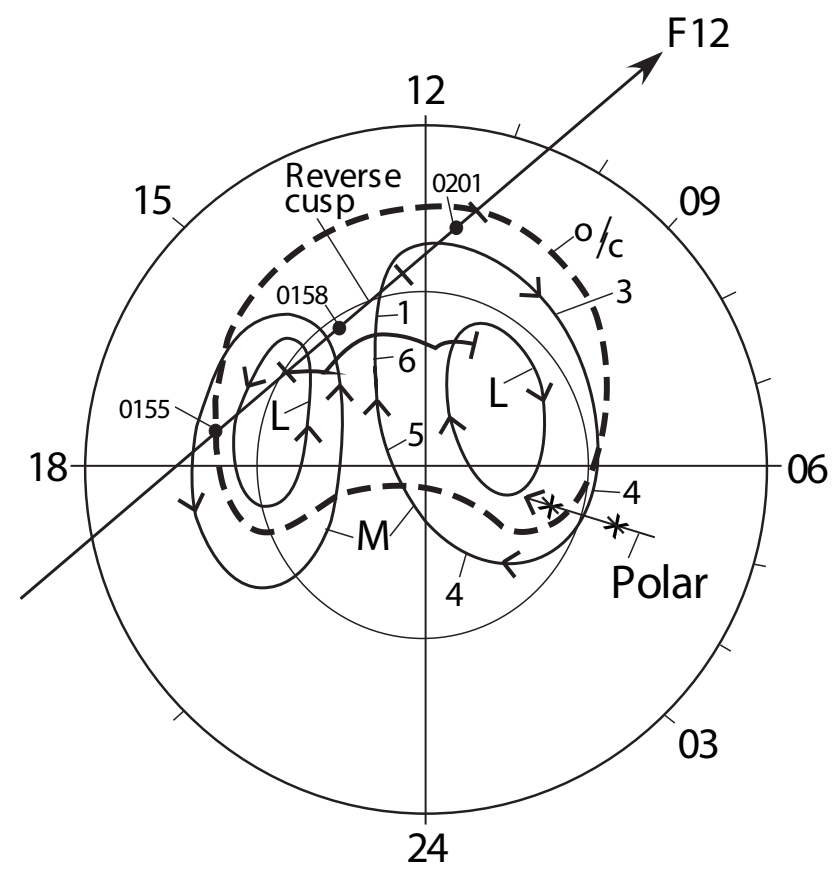

Fig. 2. Schematic illustration of convection pattern applicable to the Northern Hemisphere summer ionosphere during northeast IMF orientation $\left(B_{y}=B_{z}>0\right)$. The coordinate system is MLT-MLAT. Latitude circles representing 70 and $80^{\circ}$ MLAT are shown. Merging and lobe convection cells are marked $\mathrm{M}$ and $\mathrm{L}$, respectively. The dashed line marks the open-closed field line boundary. The ionospheric image of the type $\mathrm{C}$ reconnection in the Northern Hemisphere is shown. The footpoint of Polar is marked by star symbol at two times. The trajectory of spacecraft DMSP F12 during the interval 01:55-02:01 UT is indicated. Adapted to our case after Watanabe et al. (2005).

"reverse cusp" precipitation ("post-merging magnetosheath ions") during the interval 01:56-02:00 UT. This interval is characterized by inverse energy - latitude dispersion of precipitating ions, and sunward convection. This is the regime of the newly reconnected field lines marked 1 in the figure. The open-closed field line boundary was traversed by F12 just after 02:01 UT. The different stages of the dawn-centered convection cycle (M-cell), marked 1-6 in the present figure, correspond to the C-D cycle of Fig. 1. The L-cell corresponds to the C-cycle. The magnetic footprint of spacecraft Polar, lying in the close vicinity of the polar cap boundary in the pre-dawn sector, is indicated in the figure.

Our presentation of the Watanabe et al. (2006) model focusses on the magnetic flux circulation cycles referred to as the C- and C-D cycles with the associated ionospheric convection pattern consisting of the L- and M2 cells. The A-B cycle of Watanabe et al. (2006) (the Cowley-Crooker type) with its M1 convection cell is that which was modeled by Alexeev et al. (2008). This mode of solar windmagnetosphere coupling will be described below. 


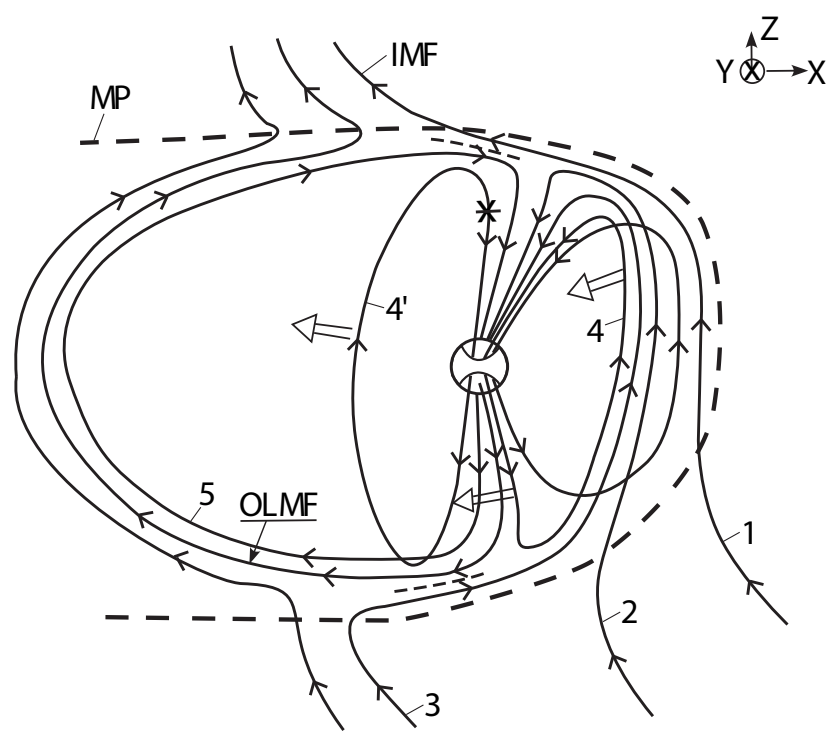

Fig. 3. Schematic illustration of magnetospheric magnetic flux cycle (marked 1-5) involving (i) high-latitude (lobe) reconnection in the north, (ii) overdraped lobe flux and (iii) internal reconnection between the overdraped lobe flux and open geomagnetic field lines in the south at the dawn side during north IMF orientation (the IMF $B_{x}<0$ case). The perspective is from the dawn side of the magnetosphere. The position of spacecraft Polar at mid-altitudes on the dawn side in relation to the field line topology are indicated by star. Adapted after Alexeev et al. (2008). Not to scale.

Figure 3 shows the flux cycle (field lines marked 2-5) described by Alexeev et al. (2008). This is the Cowley (1981) type sequential reconnection with the variant of internal reconnection occurring in both the northern and southern lobes, as suggested by Alexeev et al. (2008) and applicable to IMF $B_{z}>0, B_{x}<0$ and $B_{y}>0$ conditions. We consider the possibility of internal reconnection taking place in the Southern Hemisphere between the open lobe magnetic field (OLMF) and the overdraped lobe flux (marked 3 ) on the dawn side for the IMF $B_{y}>0$ conditions considered here. This reconnection mode give rise to the newly closed field lines marked 4 in the figure. Field line $4^{\prime}$ marks a later stage of field line 4 , after tailward convection has taken place. Field lines 2, 3 (overdraped lobe flux; see Fig. 6 of Alexeev et al. (2008)) and 4 (newly closed flux) run out of the plane of Fig. 3. The magnetic flux cycle marked 2-3-4-5 corresponds to the M1 convection cell of Watanabe et al. (2006) (see their Fig. 2). According to Watanabe et al. (2006) the intensity of the C and C-D cycles ( $\mathrm{L}-$ and $\mathrm{M} 2$ cells) are much larger than the A-B cycle (M1-cell). For the present purpose the most interesting feature is that the A-B (Cowley-Crooker-Alexeev) cycle may also give rise to newly closed field lines in the dawn sector.

A variant of the Watanabe et al. (2006) model described in Fig. 1 above, applicable to conditions of northward IMF orientation with an appreciable positive $B_{y}$ component, was elaborated by Watanabe et al. (2004). We refer to their Fig. 4. The dayside closed field lines become highly distorted with a nonzero east-west component. In this case reconnection occurs on the duskside high-latitude magnetopause in the north and on the dawnside high-latitude magnetopause in the south. In both cases the IMF reconnects with the Earth's field on the equatorward side of the cusp. The overdraped lobe fluxes on the dawn and dusk sides created by magnetopause reconnections in the north and south is called NA and NB fluxes (not shown in Fig. 1), respectively. The NB flux convects tailward and subsequently undergoes internal reconnection with closed gemagnetic flux in the southern lobe. This internal reconnection of the NB flux creates newly open and newly closed lobe fluxes on the dawn and dusk sides, respectively. The newly open field lines at dawn have the topology of field line 5 in Fig. 1 (see Fig. 4 in Watanabe et al., 2004). The resulting crescent-shaped convection cells are referred to as the primary exchange cell (PE; dusk side) and the secondary exchange cell (SE; dawn side) by Watanabe et al. (2004).

\section{Wind interplanetary observations}

Figure 4 shows plasma and magnetic field data from the SWE (Ogilvie et al., 1995) and MFI (Lepping et al., 1995) instruments on WIND for the time interval 11 August, 21:00, to 12 August 2000, 04:00 UT. From top to bottom the panels show the proton density, temperature, bulk flow speed, dynamic pressure, the total field and its components in GSM coordinates, the proton plasma $\beta$ and the Alfvén Mach number (red trace), and the IMF clock angle (i.e. the polar angle in the GSM (YZ) plane). Wind was located at $(-15,-58$, -5) $R_{E}$, while executing a so-called "petal orbit". The propagation delay Wind-subsolar magnetopause is estimated with the help of Geotail to be -1.5 min. Geotail was in the duskside magnetosheath with an average position given in GSM coordinates by $(28,13,-0.3) R_{E}$. We compare the clock angle seen by WIND and Geotail, respectively. Using the coplanarity theorem, Song et al. (1998) showed that the clock angle remains invariant across the bow shock. The bottom panel shows the two clock angle profiles overlaid (Geotail in red), where a Wind-Geotail delay of $\sim 7 \mathrm{~min}$ has been added. Good agreement may be seen. Taking now the delay time from Geotail to the subsolar magnetopause to be $5.5 \mathrm{~min}$, we arrive at a Wind-subsolar magnetopause delay of $-1.5 \mathrm{~min}$.

The interval of interest here is that between the two vertical dashed lines. It starts and ends at two field and flow discontinuities. In between the vertical lines, the magnetic field is pointing antisunward $\left(B_{x}<0\right)$, north $\left(B_{z}>0\right)$, and east $\left(B_{y}>0\right)$. The clock angle $(\theta)$ lies between $20-90^{\circ}$, and undergoes an apparent "sawtooth" variation. In the same interval many field and plasma parameters exhibit a systematic average increase (up to the UT marked by the blue line) and 


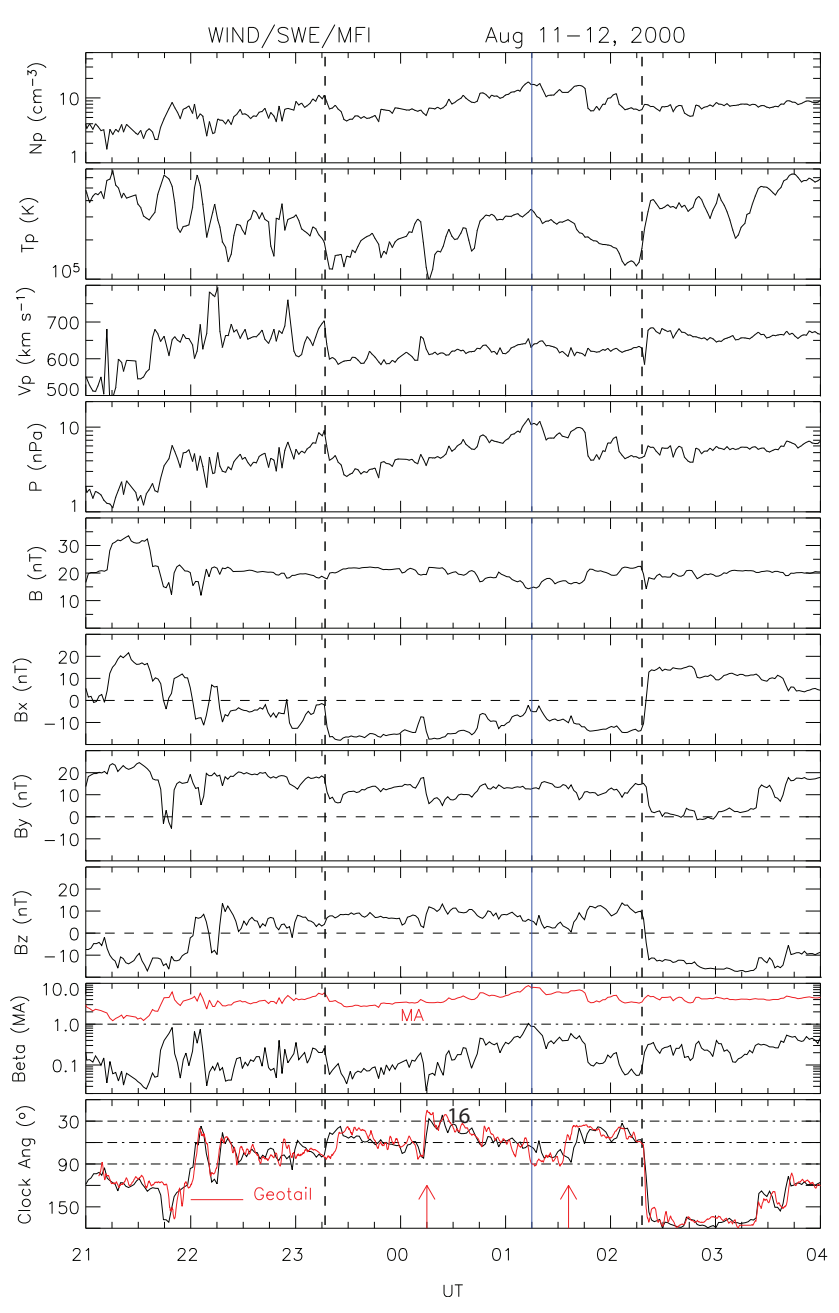

Fig. 4. Plasma and magnetic field data from spacecraft WIND for the interval 11 August, 21:00 UT, to 12 August 2000, 04:00 UT. Panels from top to bottom shows: proton density, temperature, bulk speed, dynamic pressure, total field, GSM components of the magnetic field, proton plasma $\beta$ and Alfvén Mach number (red); and the clock angle, $\theta$ (polar angle in GSM Y-Z plane).

then decrease. This trend is particularly clear in the temperature and the proton $\beta$.

The abrupt southward turning recorded by Wind at 02:20 UT is used to estimate the Wind-to-Polar propagation delay. As we shall see below the effect of this southward turning left a distinct signature in the Polar plasma observations in the magnetospheric lobe at 02:36 UT.

Figure 5 shows Wind data for the extended interval from 17:00 UT, 11 August to 23:00 UT, 13 August 2000. The same quantities are plotted as in Fig. 4. The figure shows the arrival of the shock ( $S$; first vertical guideline), the sheath region ( 19:00-06:00 UT, 11-12 August; between the first and second guidelines) and the magnetic cloud ( $\sim 06: 00-$ 22:00, 12-13 August), which is a configuration of above average magnetic field strength, large rotation of the mag-

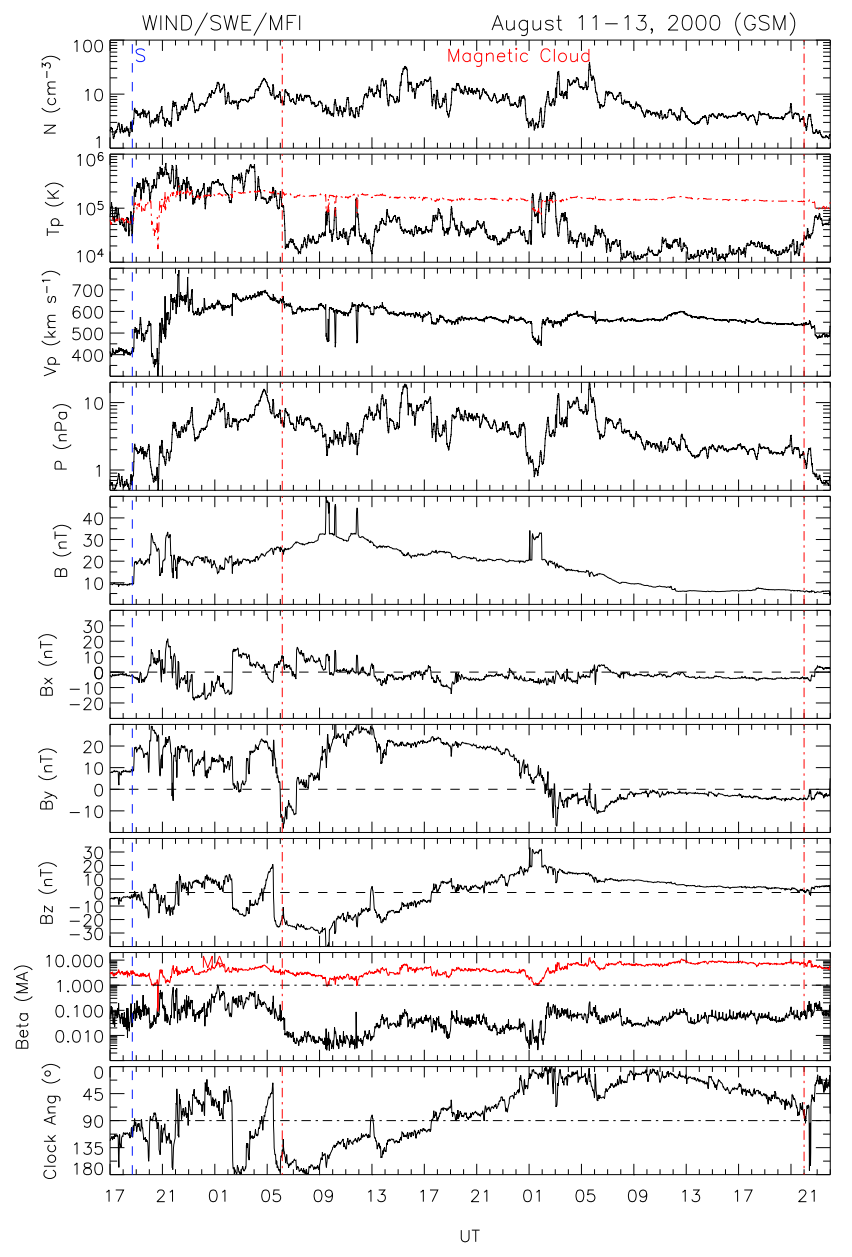

Fig. 5. Plasma and magnetic field data from spacecraft WIND for the interval 11 August (17:00 UT)-13 August (24:00 UT) 2000. Panels from top to bottom shows: proton density, temperature, bulk speed, dynamic pressure, total field, GSM components of the magnetic field, proton plasma $\beta$ and Alfvén Mach number (red); and the clock angle, $\theta$ (polar angle in GSM Y-Z plane).

netic field vector and low proton temperatures and $\beta$ (Burlaga et al., 1981). The red trace in the temperature panel gives the expected temperature for solar wind expansion after Lopez and Freeman (1986). This ejection caused a major storm $\left(D_{s t}=-237 \mathrm{nT}\right)$, one of the largest in solar cycle 23 . We work with the middle part of the sheath, clearly seen in this overview, which we expanded in the previous figure.

\section{Polar observations}

Figure 6 shows ion and electron data from Polar/HYDRA (Scudder et al., 1995) for the interval 00:0003:00 UT, 12 August 2000. The panels show from top to bottom omnidirectional ion and electron differential energy fluxes (DEFs), with intensities indicated by the respective color bars on the right, and ion DEFs flowing parallel 


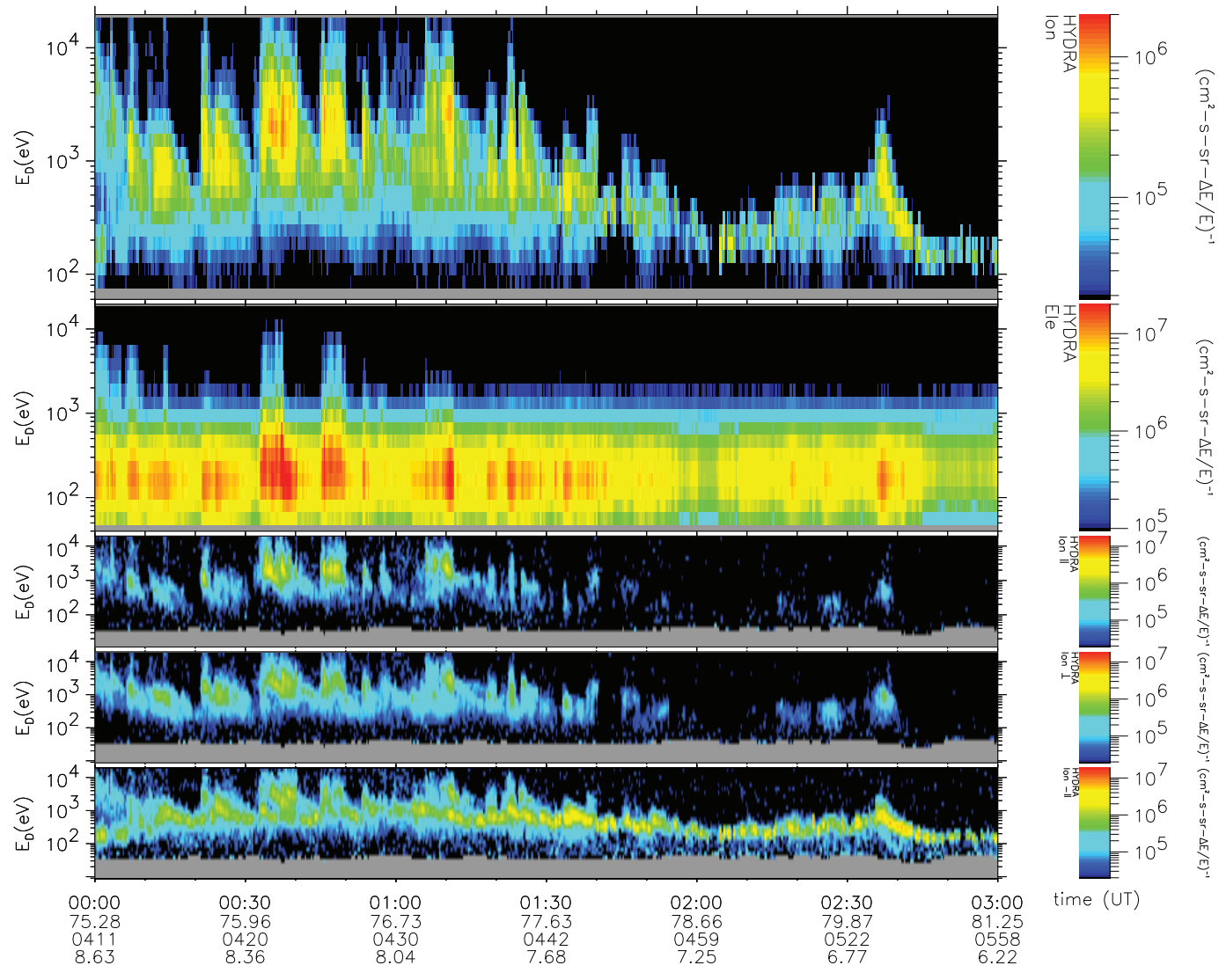

Fig. 6. Polar observations of ion and electron data for the interval 00:00-03:00 UT. Panels show: ion and electron differential energy fluxes (DEFs) as well as pitch angle (PA) restricted DEFs for the ions: parallell, perpendicular, and antiparallel to the magnetic field).

(pitch angles (PAs) between 0 and $30^{\circ}$ ), perpendicular, (PA between $75-105^{\circ}$ ), and antiparallel (PA between 150 and $180^{\circ}$ ) to the magnetic field. Positional information is shown at the bottom: UT, invariant latitude (ILT), magnetic local time (MLT) and radial distance from the Earth. The DEFs have been corrected for the effects of spacecraft potential.

Polar was ascending in latitude (ILT from $\sim 75^{\circ}$ to $81^{\circ}$ ), crossing from $\sim 04: 00$ to $\sim 06: 00 \mathrm{MLT}$, and descending in altitude (radial distance from 8.63 to $6.22 R_{E}$ ). It was situated tailward of the geomagnetic field bifurcation $\left(B_{x}>0\right.$ and $B_{z}<0$; not shown). The central feature is the bursts of ionelectron plasma throughout most of the interval. We shall focus on the following features:

1. The three strongest events within the interval 00:3001:15 UT, which are characterized by episodic enhancements of ion and electron fluxes at both low $(0.1-1 \mathrm{keV})$ and high (1-20 keV) energies. Relatively intense DEFs of ions flowing along the field are observed (panel 3) as well as somewhat lower fluxes against the field (panel 5) and tranverse to the field (panel 4). Energies of both the electron and ion fluxes extend to higher values than in the other bursts.
2. Weaker bursts of ion-electron plasma flowing predominantly against the field. The high-energy fluxes of the 00:30-01:15 UT events are lacking.

3. Electron - ion DEFs at much reduced intensities and flowing exclusively against the magnetic field as seen during the interval 01:45-02:15 UT.

4. The ion-electron event at 02:36-02:45 UT, which shows an enhancement of the flux of magnetosheath-origin plasma with ions flowing predominantly against the field (bottom panel) and a clear and protracted energylatitude dispersion of the normal type (i.e. energy decreases with increasing latitude).

5. The classical polar rain electrons and low-energy ions flowing against the field which immediately follows this dispersed signature.

Figure 7 shows Polar electron directional properties for the interval 00:00-04:00 UT. The various panels show the skew and anisotropy parameters, defined as $J_{||} / J_{-\|}(J=\mathrm{DEF})$ and $1 / 2\left[J_{||}+J_{-||}\right] / J_{\text {perp }}$, respectively (DEFs parallel, perpendicular and antiparallel to the magnetic field, and integrated (over 


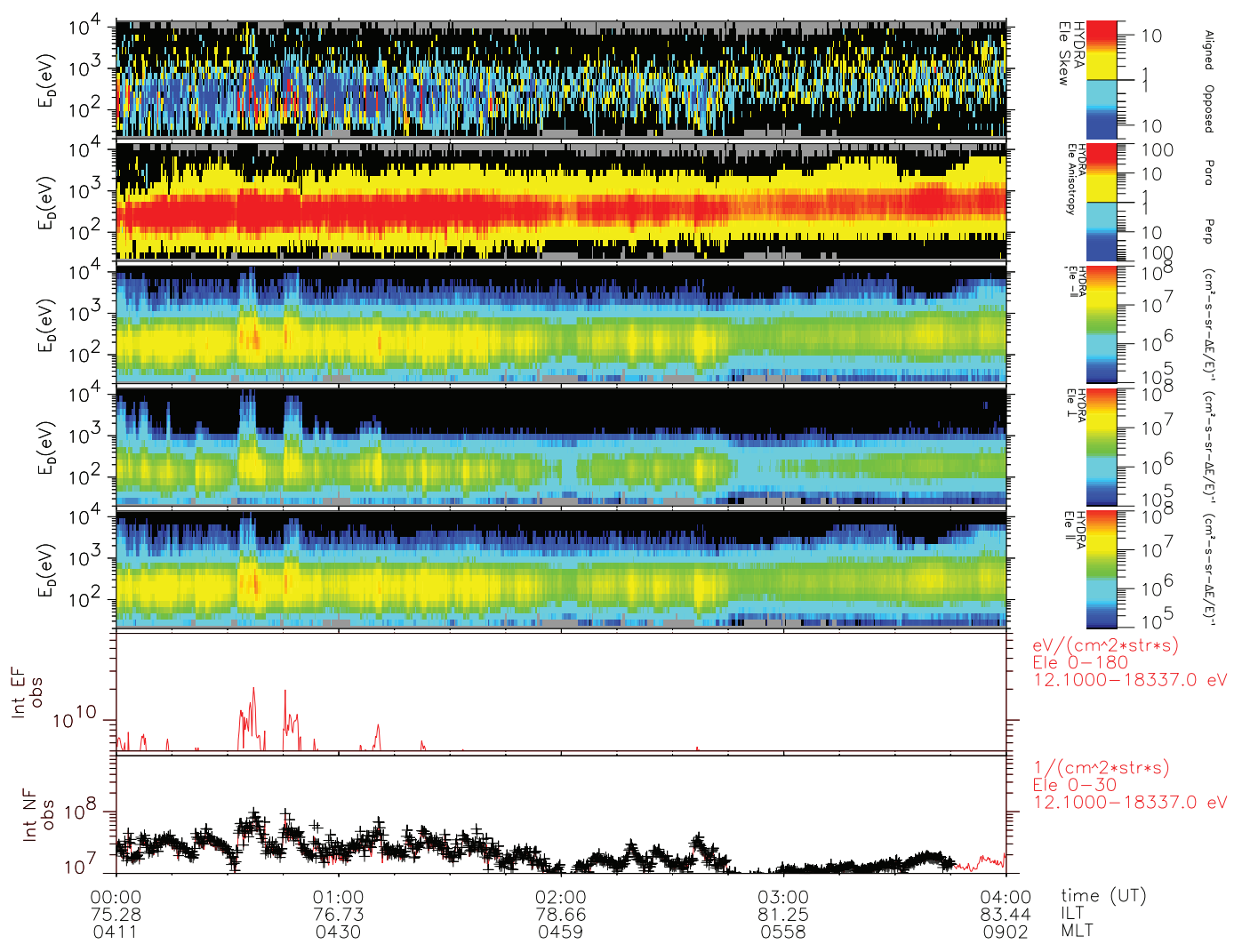

Fig. 7. Polar observations of DEFs (J) for the interval 00:00-04:00 UT. Panels from top to bottom show: (i) electron skew: $J_{\|} / J_{-\|}$, (ii) electron anisotropy:

$1 / 2\left[J_{||}+J_{-||}\right] / J_{\text {perp }}$, (iii) antiparallel flux, (iv) transverse flux, (v) parallel flux, (vi) integral energy flux, and (vii) integral number fluxes parallel and antiparallel to the field.

energy and pitch angle) energy and number fluxes.) "Parallel" is "aligned" and antiparallel is "opposed". In the last panel red and black symbols refer to electron fluxes parallel and antiparallel to the field. We shall focus on the three major flux enhancements observed during the interval 00:3001:15 UT. Here the electrons are predominantly streaming along the field (see panel 2) with comparable parallel and antiparallel fluxes, i.e. a bi-directionally streaming electron population up to $\sim 10 \mathrm{keV}$ energies. This property of the electron flux is often taken as an indication of closed field lines (see e.g. Chang et al., 1998; Lavraud et al., 2006).

Figure 8 shows densities during the Polar ion bursts (second panel) and the clock angle of the sheath field (top panel) for the interval 00:00-03:00 UT. Four subintervals 1-4 are identified by a combination of clock angle regime and the properties of the ion-electron bursts. The arrow marks the last intense ion-electron event commencing at 02:36 UT. As noted, we used this event to estimate the Wind-to-Polar propagation delay which is the sum of (i) the time from Wind to the subsolar magnetopause $(-1.5 \mathrm{~min})$ and (ii) the "intrinsic" delay from the subsolar magnetopause to Polar. The normal polar rain took over after the 02:36-02:42 UT event. This

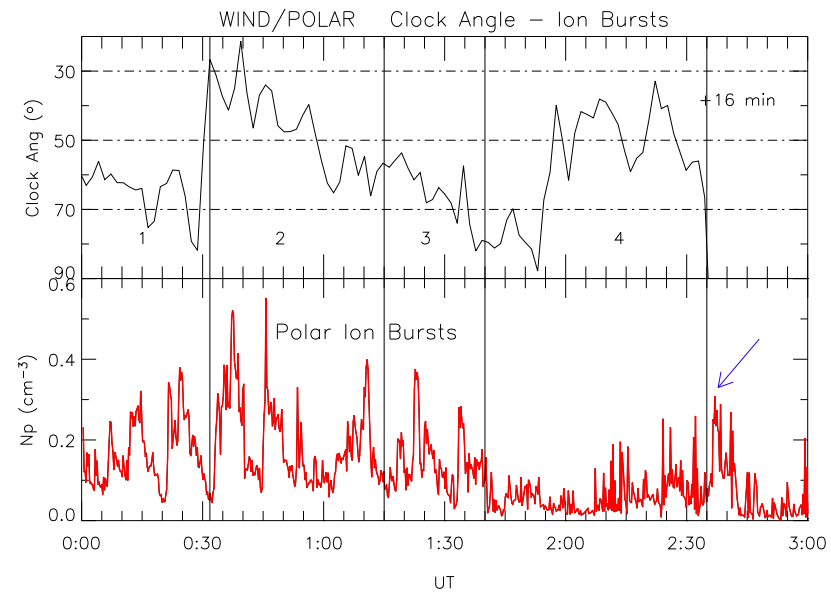

Fig. 8. Top panel: Clock angle at Wind shifted forward by $16 \mathrm{~min}$, our estimated Wind-Polar propagation delay. Bottom: Polar densities corresponding to the ion bursts. Numbered subintervals are delimited by vertical guidelines. 


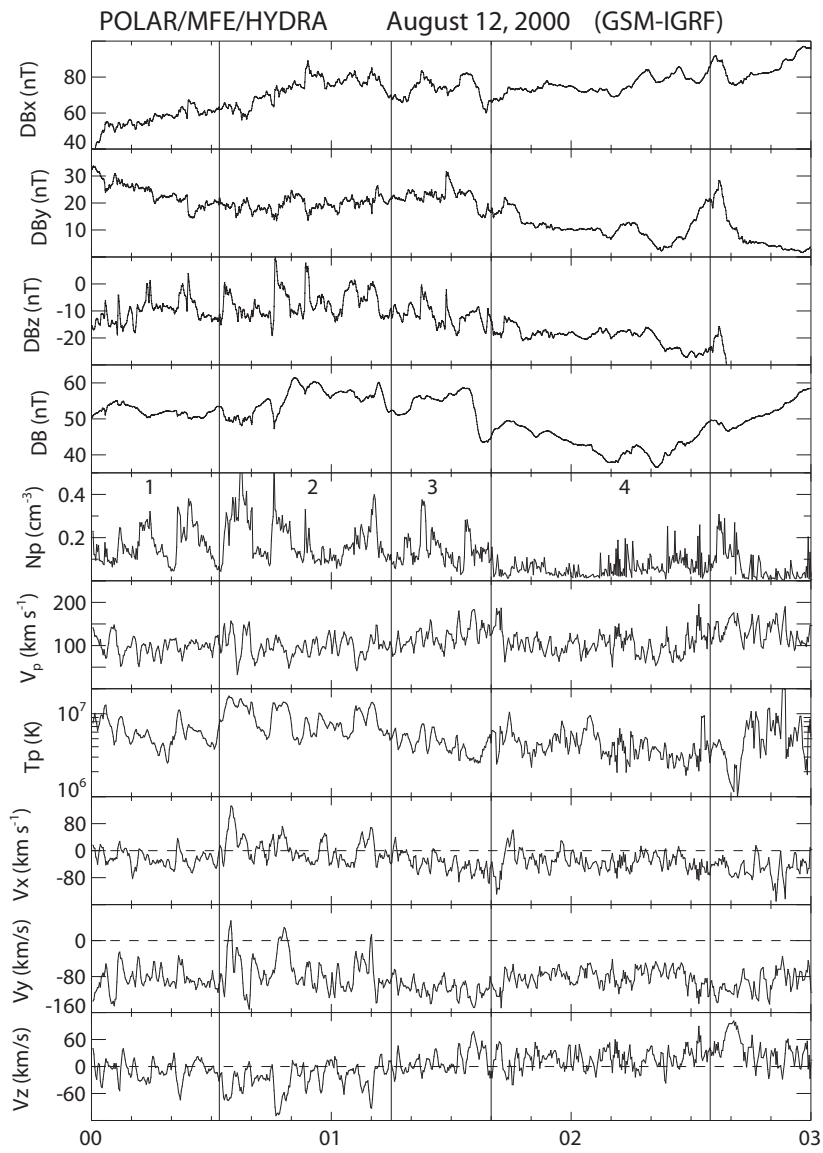

Fig. 9. Polar observations of magnetic deflections, plasma density, bulk speed, and the ion velocity components $V_{x}, V_{y}$, and $V_{z}$ shown in GSM coordinates. Subintervals are marked by vertical guidelines.

suggests that the Wind sheath field data should be delayed by approximately $16 \mathrm{~min}$ so that the 02:36-02:42 UT event and the normal polar rain intervals respond to the sharp southward turning and the subsequent interval of strongly south IMF orientation (Fig. 4). Correspondingly, a 16 min time delay is added to the Wind time in this figure, and it is this time delay we shall use when relating the ion/electron bursts to the clock angle.

From this estimate we infer the following association between the clock angle at Wind and the ion-electron bursts recorded by Polar. (i) the two first bursts (interval 1) occurred at $\theta \sim 60^{\circ}$, (ii) the three most intense bursts (interval 2): $\theta \sim 30-60^{\circ}$, (iii) less intense ion bursts in interval 3: $\sim 60-70^{\circ}$, (iv) the period when ion energies decrease and the bursts are weaker (interval 4): fairly small clock angles, (v) the 02:36-02:42 UT burst (marked by arrow) initiated by the rapid southward turning, (vi) low ion fluxes and polar rain electrons after 02:42 UT: large $\left(>90^{\circ}\right)$ clock angles.

Figure 9 includes information on the magnetic field perturbations detected by the POLAR/MFE instrument (Russell,
1995). The figure displays the magnetic perturbations (with respect to the IGRF reference field) in the $\mathrm{x}, \mathrm{y}$, and $\mathrm{z}$ components, the difference between the measured field strength and the model field, and then the proton density, bulk speed, temperature, and GSM components of the flow vector. Vertical lines mark the four intervals into which we have subdivided the event. The intense ion bursts (marked by density enhancements) are accompanied by magnetic deflections, most evident in the $B_{z}$ component, and by perturbations in all three components of the velocity vector. The three major events in interval 2 are noteworthy in the following respects. They show up as strong velocity deflections with respect to the background level, characterized by positive $V_{x}$, positive $V_{y}$, and negative $V_{z}$. They are also marked by positive $B_{z}$ deflections (unipolar, box-car profile). The bursts in interval 2 (00:32-01:15 UT) are also accompanied by enhanced temperatures. (This is the interval of major interest for this work.) There are smaller field deflections in interval 3, none in interval 4 , but flow perturbations are much reduced in both.

The dispersed signature at 02:35-02:45 UT shows a very different signature: a strong, positive $V_{z}$ deflection combined with a significant drop in ion temperature. This is the opposite signature to that in the interval 2 events. This difference in the plasma characteristics will be explained below in terms of newly closed versus old open field lines in the magnetospheric lobe.

\section{Interpretation}

We have reported Polar observations of ion-electron fluxes, ion drifts and local magnetic deflections at mid magnetospheric altitudes $\left(8-7 R_{E}\right)$ during a Northern Hemisphere pass in the pre-dawn sector of the polar cap boundary. These plasma/field observations on field lines of the magnetospheric Northern Hemisphere lobe are divided in different categories (subintervals) sorted by the orientation of the magnetic field in the sheath of the magnetic cloud. The observations will be interpreted in terms of different possible topologies of solar wind-magnetosphere coupling in the midaltitude lobes of the Northern Hemisphere, laying emphasis on the changes of the lobe plasma (ion-electron bursts) detected by Polar under the variable magnetosheath magnetic field conditions detected by spacecraft Wind.

A central issue is to inquire if internal reconnection was present at the dawn flank, and if so, which mode of internal reconnection occurred in our case. According to the model proposed by Watanabe et al. (2004) for oblique northward IMF (clock angles around $45^{\circ}$; IMF $B_{y}>0$ ) we should expect newly-open field lines at the dawn flank (field line marked 3 in their Fig. 4b) resulting from reconnection of overdraped lobe flux and the closed geomagnetic field lines. The associated convection cell is the crescent-shaped SE cell of Watanabe et al. (2004) (see their Fig. 17). The reason newly-closed flux would not be expected in the Watanabe et al. (2004) 
scenario is that newly-closed flux forms on the opposite side of the magnetosphere.

In the model proposed by Watanabe et al. (2006), applicable to strongly northward IMF conditions and significant dipole tilt and/or IMF $B_{x}$ component, we would expect both newly-closed (4) and newly-open (5) field lines with postmerging ions of magnetosheath origin on the dawn flank (see their Fig. 2a). This is the result of internal reconnection of overdraped lobe flux and the closed field of the Earth (type D reconnection of Watanabe) as indicated in Fig. 1. The associated convection cell is the "reverse" $M_{2}$-cell shown in Fig. 2 of Watanabe et al. (2006) and adapted to our case in Fig. 2 in the present paper.

The third option we consider is the newly closed flux at dawn excited by internal reconnection between two open field lines as described in Fig. 3. When two open field lines reconnect to form a newly closed field line we have the phenomenon of "capture" of magnetosheath plasma by the magnetosphere. Thus, this type of newly closed field line (4 in Fig. 3) ought to contain plasma with magnetosheath properties, i.e. cold and dense. In contrast, line 4 in Fig. 1, created by reconnection of an open and a closed field line, will entrap magnetospheric plasma previously residing on the closed field line in addition to magnetosheath plasma. Its energy should be higher (higher $T_{p}$ ).

Since Polar was in the right place to detect newly-open and/or newly-closed lobe fluxes at dawn, and given that the observations are made under dipole tilt (12 August), we are in a position to discriminate between the three scenarios described above.

Our interval 2 (clock angle range: $30-60^{\circ}$ and $B_{x} \leq-10 \mathrm{nT}$ ) is characterized by the three major ion-electron bursts showing (i) high fluxes of magnetosheath electrons and ions, (ii) the presence of a high-energy tail in the electron and ion fluxes, (iii) high-energy component of the electron flux consisting of bi-directionally streaming electrons of plasma sheet origin (trapped population), (iv) a negative $V_{z}$ flow component, and (iv) ion temperature enhancement. This is a mixture of plasmas of magnetosheath and magnetosphere origins which is typical of newly-closed magnetic flux. On this point we refer to the similar observations of Bogdanova et al. (2007). They noted the following plasma characteristics of the newly closed fluxes:

1. compared to the observations in the open LLBL the whole population of ions is shifted towards higher energies,

2. strong sunward convection up to $50 \mathrm{~km} / \mathrm{s}$ is observed,

3. the electron population is a mixture of bi-directional magnetosheath and trapped plasma sheet,

4. ion data indicates dual reconnection operating not in steady state, but in the pulsed regime, and
5. LLBL/cusp is formed in the dawn sector of the magnetosphere and does not convect from the dayside.

We find that the Polar observations in our interval 2 are consistent with features (1), (3), (5) and possibly (4) from the Bogdanova case.

Contrary to the directional properties of the ion fluxes of the other intervals, a strong component of ion fluxes is flowing along the field in interval 2. This is reflected in the $V_{z}$ negative ion flow events in Fig. 9. We interpret the ion-electron bursts in interval 2 to be the result of plasma capture on field lines that have been newly-closed by the type D reconnection (i.e. internal reconnection in the Southern Hemisphere between overdraped lobe flux and closed magnetospheric field lines) of Watanabe et al. (2006) (field line marked 4 in Fig. 1). This scenario explains the mixture of the magnetosheath type and the higher-energy magnetospheric component of the ion fluxes in interval 2.

The bi-directionally streaming electrons is often used as an indicator of closed field lines. The observation of bidirectional magnetosheath electron fluxes (see our Fig. 7) has been used to infer the presence of bursty events of newlyclosed field lines under similar IMF conditions as in our case (northward IMF with significant $B_{y}$ component) by Bogdanova et al. (2007) and Zong et al. (2008). We note that magnetosheath electrons are not bi-directional in general. They become so after the field line is closed, as a result of reflection in the stronger fields at the ionospheric part of the field lines. Bi-directional electrons are often seen in the LLBL.

From the Polar data in interval 2 we infer the temporal structure of the two-step reconnection process to be (i) pulsed (as in the Bogdanova et al. case) or (ii) time-varying continuous reconnection. A third possibility would be that the spacecraft is moving in and out of a steady-state reconnection region. The latter option we consider to be less likely in view of the relatively steady (or continuously changing) IMF and solar wind conditions in interval 2.

Our intervals 1 and 3 (clock angle $=60-80^{\circ}$ ) show ion fluxes flowing mainly against the field. The high-energy component of interval 2 events is missing. Since this is observed during times when the clock angle of the external field is $>60^{\circ}\left(\left|B_{y} / B_{z}\right|>1\right)$ the model of Watanabe et al. (2004) (oblique northward IMF) may be applicable in this case. In that case the ion bursts are located on the recently open lobe field lines marked 3 in Fig. 4b of Watanabe et al. (2004). Newly open field lines are excluded due to the less intense ion fluxes flowing along the field. However, these data may also fit with the other options, such as the Watanabe et al. (2006) scenario (field line 5 in Fig. 1).

The less intense and less energetic ion fluxes flowing antiparallel to the field in our later interval 4 (clock angle $=40$ $60^{\circ}$ ) are interpreted as mirrored ions on old open lobe flux created by lobe reconnection in the Northern Hemisphere, i.e. the "uncoupled type $\mathrm{C}$ reconnection" (field lines marked 
1, 2 and 6 in Fig. 1), after Polar has moved to higher MLATs in the polar cap (see Fig. 2). We note that these NA fluxes should be present in our case since lobe reconnection in the Northern Hemisphere is favoured by both the prevailing negative $B_{x}$ component of the sheath field and the summer season.

Our 02:36-02:45 UT dispersed signature (clock angle $=160-180^{\circ}$ ) is an isolated event having enhanced fluxes of magnetosheath origin plasma flowing totally against the field. These are mirrored ions on old open field lines created by reconnection at low dayside latitudes and threading the Polar position during strongly southward directed sheath field of the type which has been documented previously by Lockwood et al. (2001). The event is initiated by the rapid southward turning of the sheath field recorded by Wind at 02:15 UT. It represents a marker of when the reconnection mode changed and its clear characteristics furnished us with a timer to lag the IMF from Wind to Polar (see Fig. 8).

The classical polar rain fluxes in the following interval is that which is typical of the hemisphere favoured by IMF $B_{x}$ (Fairfield and Scudder, 1985). It is consistent with old open flux created by steady state dayside magnetopause reconnection under the stable condition of a strongly south directed sheath field.

The association of the ion-electron bursts with magnetic deflections (see Fig. 9), and the location of Polar (tailward of the magnetospheric field bifurcation and at high latitudes) lead us to think that these events are being observed on magnetic flux tubes excited by post-terminator FTEs. The field perturbations manifested themselves as a reversal in the polarity of GSM $B_{z}$ with respect to the background field. These imply a confined current system similar to that ascribed to high-latitude FTEs reported by Thompson et al. (2004), but for northward IMF in our case. According to Kawano and Russell (1997) lobe (cusp) reconnecion is one of different possible sources of post-terminator FTEs. The consequence of our interpretation is that the internal reconnection of the overdraped lobe flux (the type $\mathrm{D}$ reconnection of Watanabe et al., 2006) may be another source of post-terminator FTEs.

\section{Summary and conclusions}

We have reported observations of a sequence of bursts of ion-electron plasma in the Northern Hemisphere magnetospheric lobe, just tailward of the dawn-dusk terminator during the passage at Earth of a magnetic cloud sheath whose (strong) magnetic field was directed northward. The clock angle of the cloud sheath field was in the range 20 to 90 degrees. We could isolate an evolution of the fluxes at Polar - in terms of energy and flow direction of the particles - and relate this to changes in the cloud sheath clock angle and the $B_{x}$ component. An example of the evolution of the ion flux is the change of energy range spanned by the plasma bursts, from $0.2-2 \mathrm{keV}$ to $1-10 \mathrm{keV}$ that occurred between 00:15-00:35 UT (Fig. 6). Corresponding transitions in the reconnection geometry were inferred, which were governed by the changes in the medium in which the magnetosphere is immersed.

The particle fluxes consisting of a mixture of particles of magnetosheath and magnetosphere origin plasmas with a trapped high-energy component are interpreted in terms of plasma captured on newly-closed lobe field lines after internal reconnection with geomagnetic field lines near dawn in the winter, Southern Hemisphere. The bursty nature of the events and the associated local magnetic deflections suggest a relationship with post-terminator FTEs. The observations are discussed in relation to three possible scenarios of magnetospheric magnetic flux circulation that may be realized in our case (Watanabe et al., 2004, 2006). The three models put emphasis on slightly different aspects of solar wind - magnetosphere coupling where the different features of the external conditions, such as the $B_{x}$ and $B_{y}$ components, and dipole tilt, play important roles.

The A-B cycle described in Fig. 3 involves newly closed flux on the dawn flank excited by internal reconnection of two open field lines. When two open field lines reconnect to form a newly closed field line we have the phenomenon of "capture" of magnetosheath plasma by the magnetosphere. Thus, this type of newly closed field line (marked 4 in Fig. 3) ought to contain plasma with magnetosheath properties, i.e. cold and dense. In contrast, field line 4 in Fig. 1, created by reconnection of an open overdraped lobe field line and a closed geomagnetic field line, will entrap magnetospheric plasma previously residing on the closed field line in addition to magnetosheath plasma. Its energy should be higher (higher $T_{p}$ ), and that's what we see in our interval 2.

Thus, a main focus in this paper is the discrimination between the reconnection scenarios proposed by Watanabe et al. (2004) and Watanabe et al. (2006). Watanabe et al. (2006) refer to a situation where the IMF is due north. In this case reconnections occur sequentially in different hemispheres (first at the magnetopause in the hemisphere favoured by $B_{x}$ polarity/season and then inside the magnetosphere in the other hemisphere). In the Watanabe et al. (2004) model a nonzero IMF $B_{y}$ component is essential in creating the interconnection topology. In this case sequential reconnections occur at the magnetopause and inside the magnetosphere in the same hemisphere. The two models describe completely different reconnection configurations and different, specific convection cycles are predicted. The two models deal with two different sources of overdraped lobe flux.

The Watanabe et al. (2004) model, applicable to oblique northward IMF with an appreciable $B_{y}$ component, may be relevant to our case: $B_{y}=8-15 \mathrm{nT}$. Overdraped lobe flux (the NB flux) on the dusk side resulting from magnetopause reconnection in the Southern Hemisphere is subsequently reconnected with closed field lines (internal reconnection) in 
the south. This results in newly-closed flux at dusk and newly open flux at dawn. The latter may be represented by field line 5 in Fig. 1. The indicated magnetic flux cycles correspond to crescent-shaped convection cells at dawn and dusk.

The model of Watanabe et al. (2006), applicable to strongly northward IMF conditions with dipole tilt and/or nonzero IMF $B_{x}$, is also relevant for our case: $B_{x}=-10 \mathrm{nT}$ and mid-August. Overdraped lobe flux on the dawn side resulting from magnetopause reconnection with the Northern Hemisphere lobe field (type $\mathrm{C}$ reconnection favoured by $B_{x}$ polarity) is subsequently reconnected (internal reconnection) with closed field lines in the south (type D reconnection). Newly closed flux appears at dawn (field line 4 in Fig. 1). A multi-phase convection cycle of the "reverse" M2-cell type (newly open lobe - overdraped lobe - newly-closed lobe re-opened lobe) is expected (see Figs. 1 and 2).

As detailed above we find evidence of the Watanabe et al. (2006) model in the Polar observations in our interval 2 , restricted to relatively small clock angles $\left(\leq 60^{\circ}\right)$ and $B_{x} \leq-10 \mathrm{nT}$, indicating plasma injections from two different sources: the closed field regime of the magnetosphere and the magnetosheath. The presence of a high-energy component of trapped plasma (bi-directionally streaming electrons at $1-10 \mathrm{keV}$ ) mixed with the magnetosheath-origin fluxes is evidence of newly-closed field lines at dawn (marked 4 in Fig. 1) excited by internal reconnection of overdraped lobe flux and the closed field of the Earth. Thus in this particular case (our interval 2) it seems that the dipole tilt (midAugust) and the $B_{x}$ component $(-10 \mathrm{nT})$ of the cloud sheath field in combination with the low clock angles (and $B_{y}>0$ ) were decisive factors for the realization of this lobe reconnection mode. The possible association with postterminator FTEs furthermore indicates that they are effective in capturing magnetosheath plasma on closed field lines in the dawn flank LLBL during IMF $B_{x}<0, B_{y}>0$, and $B_{z}>0$ conditions.

Acknowledgements. This work is supported by NASA grants WIND/SWE and MFI Analysis, NNX08AD11G and NAG5NNG05GG25G. It is also supported by the Norwegian Research Council and AFOSR Task 2311AS. Access to the DMSP data base (https://swx.plh.af.mil) was kindly provided by W. F. Denig.

Topical Editor I. A. Daglis thanks N. Crooker and another anonymous referee for their help in evaluating this paper.

\section{References}

Alexeev, I. I., Belenkaya, E. S., Bobrovnikov, S. Y., Kalagaev, V. V., Cumnock, J. A., and Blomberg, L. G.: Magnetopause mapping of the ionosphere for northward IMF, Ann. Geophys., 25, 26152625, 2008 http://www.ann-geophys.net/25/2615/2008/.

Bogdanova, Y. V., Owen, C. J., Siscoe, G., Fazakerley, A. N., Dandouras, I., Marghitu, O., Kaymaz, Z., Reme, H., and Lucek, E. A.: Cluster observations of the magnetospheric lowlatitude boundary layer and cusp during extreme solar wind and interplanetary magnetic field conditions: II. 7 November
2004 ICME and statistical survey, Solar Phys., 244, 233-261, doi:10.1007/s11207-007-0418-0, 2007.

Burlaga, L. F., Sittler, E., Mariani, F., and Schwenn, R.: Magnetic loop behind an interplanetary shock: Voyager, Helios, and IMP8 observations, J. Geophys. Res., 86, 6673-6684, 1981.

Chang, S. W., Scudder, J. D., Sigwarth, J. B., Frank, L. A., Maynard, N. S., Burke, W. J., Peterson, W. K., Shelley, E. G., Friedel, R., Blake, J. B., Greenwald, R. A., Lepping, R. P., Sofko, G. J., Villain, J. P., and Lester, M.: A comparison of a model for the theta aurora with observations from Polar, J. Geophys. Res., 103, 17367-17390, 1998.

Chisham, G., Freeman, M. P., Coleman, I. J., Pinnock, M., Hairston, M. R., Lester, M., and Sofko, G.: Measuring the dayside reconnection rate during an interval of due northward interplanetary magnetic field, Ann. Geophys., 22, 4243-4258, 2004, http://www.ann-geophys.net/22/4243/2004/.

Cowley, S. W. H.: Magnetospheric and ionospheric flow and the interplanetary magnetic field, in: The Physical Basic of the Ionosphere in the Solar-Terrestrial System, no. 295 in Conference Proceedings, pp. 4-1-4-12, AGARD (Advisory Group for Aerospace Research \& Development), NATO, Neuilly sur Seine, France, 1981.

Crooker, N.: Reverse convection, J. Geophys. Res., 97, 19363 19372, 1992.

Fairfield, D. H. and Scudder, J. D.: Polar rain: Solar coronal electrons in the Earth's magnetosphere, J. Geophys. Res., 90, 40554068, 1985.

Imber, S. M., Milan, A. E., and Hubert, B.: The auroral and ionospheric flow signatures of dual lobe reconnection, Ann. Geophys., 24, 3115-3129, 2006, http://www.ann-geophys.net/24/3115/2006/.

Kawano, H. and Russell, C. T.: Cause of postterminator flux transfer events, J. Geophys. Res., 102(A12), 27 029-27 038, 1997.

Lavraud, B., Thomsen, M. F., Lefevre, B., Schwatrtz, S. J., Seki, K., Phan, T. D., Wang, Y. L., Fazakerley, A., Reme, H., and Balogh, A.: Evidence for newly closed magnetosheath field lines at the dayside magnetopause under northward IMF, J. Geophys. Res., 111, A05211, doi:10.1029/2005JA011266, 2006.

Lepping, R. P., Acũna, M. H., Burlaga, L. F., Farrell, W. M., Slavin, J. A., Schatten, K. H., Mariani, F., Ness, N. F., Neubauer, F. M., Whang, Y. C., Byrnes, J. B., Kennon, R. S., Panetta, P. V., Scheifele, J., and Worley, E. M.: The WIND magnetic field investigation, Space Sci. Rev., 71, 207-229, 1995.

Lockwood, M., Milan, S. E., Onsager, T., Perry, C. H., Scudder, J. A., Russell, C. T., and Brittnacher, M.: Cusp ion steps, fieldaligned currents and poleward moving auroral forms, J. Geophys Res., 106, 29 555-29 570, 2001.

Lopez, R. E. and Freeman, J. W.: Solar wind proton temperature velocity relationship, J. Geophys. Res., 91, 1701-1705, 1986.

Ogilvie, K. W., Chornay, D., Fritzenreiter, R., Hunsaker, F., Keller, J., Lobell, J., Miller, G., Scudder, J. D., Sittler Jr., E. C., Torbert, R. B., Bodet, D., Needell, G., Lazarus, A. J., Steinberg, J. T., Tappan, J. H., Mavretic, A., and Gergin, E.: SWE, a comprehensive plasma instrument for the WIND spacecraft, Space Sci. Rev., 71, 55-77, 1995.

Russell, C. T.: The GGS/Polar magnetic field investigation, vol. 71 of Space Sci. Rev., Kluwer Academic Publishers, 1995.

Scudder, J., Hunsacker, F., Miller, G., Lobell, J., Zawistowski, T., Ogilvie, K., Keller, J., Chornay, D., Herrero, F., Fitzenreiter, R., 
Fairfield, D., Needell, J., Bodet, D., Googins, J., Kletzing, C., Torbert, R., Vandiver, J., Bentley, R., Fillius, R., McIlwain, C., Whipple, E., and Korth, A.: Hydra - A 3-dimensional electron and ion hot plasma instrument for the Polar spacecraft of the GGS mission, Space Sci. Rev., 71, 459-495, 1995.

Song, P., Russell, C. T., and Thomsen, M. F.: Slow mode transition in the frontside magnetosheath, J. Geophys. Res., 97, 82958305, 1998.

Tanaka, T.: Configuration of the magnetosphere-ionosphere convection system under northward IMF conditions with nonzero IMF $B_{y}$, J. Geophys. Res., 104, 14 683-14 690, 1999.

Thompson, S. M., Kivelson, M. G., Khurana, K. K., Balogh, A., Reme, H., Fazakerley, A. N., and Kistler, L. M.: Cluster observations of quasi-periodic impulsive signatures in the dayside northern lobe: High-latitude flux transfer events?, J. Geophys. Res., 109, A02213, doi:10.1029/2003JA010138, 2004.

Watanabe, M., Sofko, G., and Andre, D. A.: Polar cap bifurcation during steady-state northward interplanetary magnetic field with $\left|B_{y}\right| \sim B_{z}$, J. Geophys. Res., 109, A01215, doi:10.1029/2003JA009944, 2004.
Watanabe, M., Kabin, G., Sofko, G. J., Rankin, R., Gombosi, T. I., Ridley, A., and Clauer, C. R.: Internal reconnection for northward interplanetary magnetic field, J. Geophys. Res., 110, A06210, doi:10.1029/2004JA010832, 2005.

Watanabe, M., Sofko, G. J., Andre, D. A., Ruohoniemi, M., Hairston, M. R., and Kabin, K.: Ionospheric signatures of internal reconnection for northward interplanetary magnetic field: Observations of "reciprocal cells" and magnetosheath ion precipitation, J. Geophys. Res., 111, A06201, doi:10.1029/2005JA011446, 2006.

Zong, Q.-G., Zhang, H., Fritz, T. A., Goldstein, M. L., Wing, S., Keith, W., Winningham, J. D., Frahm, R., Dunlop, M. W., Korth, A., Daly, P. W., Reme, H., Balogh, A., and Fazakerly, A. N.: Multiple cusps during an extended northward IMF period with a significant $B_{y}$ component, J. Geophys. Res., 113, A01210, doi:10.1029/2006JA012188, 2008. 